
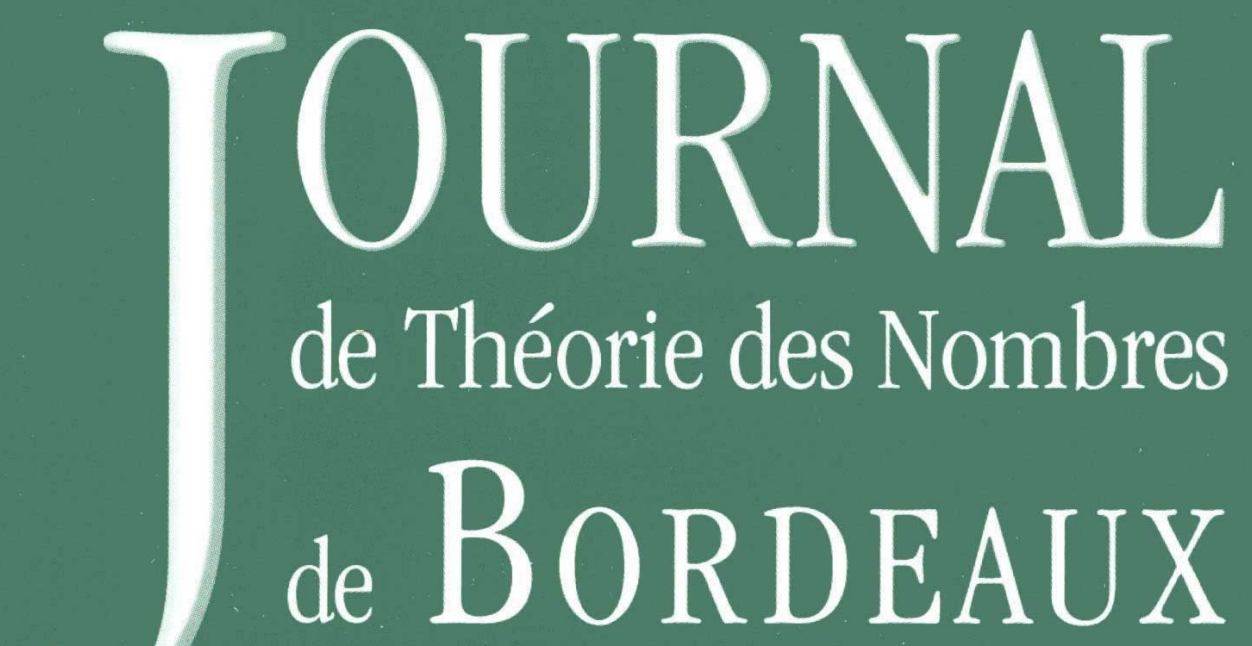

anciennement Séminaire de Théorie des Nombres de Bordeaux

\title{
Karin HALUPCZOK
}

On the ternary Goldbach problem with primes in arithmetic progressions having a common modulus

Tome 21, no 1 (2009), p. 203-213.

<http://jtnb.cedram.org/item?id=JTNB_2009__21_1_203_0>

(C) Université Bordeaux 1, 2009, tous droits réservés.

L'accès aux articles de la revue «Journal de Théorie des Nombres de Bordeaux » (http://jtnb.cedram.org/), implique l'accord avec les conditions générales d'utilisation (http://jtnb.cedram. org/legal/). Toute reproduction en tout ou partie cet article sous quelque forme que ce soit pour tout usage autre que l'utilisation à fin strictement personnelle du copiste est constitutive d'une infraction pénale. Toute copie ou impression de ce fichier doit contenir la présente mention de copyright.

\section{cedram}




\title{
On the ternary Goldbach problem with primes in arithmetic progressions having a common modulus
}

\author{
par KARIN HALUPCZOK
}

\begin{abstract}
RÉSumÉ. Pour $\varepsilon>0$ et $n$ impair suffisamment grand, nous montrons que, pour presque tout $k \leq R:=n^{1 / 5-\varepsilon}$, il existe une représentation $n=p_{1}+p_{2}+p_{3}$ avec des nombres premiers $p_{i} \equiv b_{i}$ modulo $k$ pour presque tout triplet admissible $b_{1}, b_{2}, b_{3}$ de résidus modulo $k$.
\end{abstract}

ABSTRACT. For $\varepsilon>0$ and any sufficiently large odd $n$ we show that for almost all $k \leq R:=n^{1 / 5-\varepsilon}$ there exists a representation $n=p_{1}+p_{2}+p_{3}$ with primes $p_{i} \equiv b_{i} \bmod k$ for almost all admissible triplets $b_{1}, b_{2}, b_{3}$ of reduced residues $\bmod k$.

\section{Introduction and results}

Let $n$ be a sufficiently large integer, consider an integer $k$ and let $b_{1}, b_{2}, b_{3}$ be integers that are relatively prime to $k \geq 1$, with $0 \leq b_{i}<k, i=1,2,3$.

We consider the ternary Goldbach problem of writing $n$ as

$$
n=p_{1}+p_{2}+p_{3}
$$

with primes $p_{1}, p_{2}$ and $p_{3}$ satisfying the three congruences

$$
p_{i} \equiv b_{i} \bmod k, i=1,2,3
$$

for the common modulus $k$. One is interested in the solvability of this question for all sufficiently large $n$ with the modulus $k$ being as large as some power of $n$. This problem has been studied intensely by many authors. For an overview, see for example [2].

An obvious necessary condition for solvability is

$$
n \equiv b_{1}+b_{2}+b_{3} \bmod k
$$

as otherwise no such representation of $n$ is possible.

We call such a triplet $b_{1}, b_{2}, b_{3}$ of reduced residues mod $k$ admissible, and a pair $b_{1}, b_{2}$ of reduced residues admissible, if $\left(n-b_{1}-b_{2}, k\right)=1$. For a given $b_{1}$ we call $b_{2}$ admissible, if $b_{1}, b_{2}$ is an admissible pair. Let us denote the number of these admissible pairs respectively triplets by $A(k)$. 
We make our consideration of this strengthened ternary Goldbach problem more precise in the following way. Let

$$
J_{3}(n):=J_{k, b_{1}, b_{2}, b_{3}}(n):=\sum_{\substack{m_{1}, m_{2}, m_{3} \leq n \\ m_{1}+m_{2}+m_{3}=n \\ m_{i} \equiv b_{i}(k), i=1,2,3}} \Lambda\left(m_{1}\right) \Lambda\left(m_{2}\right) \Lambda\left(m_{3}\right),
$$

where $\Lambda$ is von Mangoldt's function. The function $J_{3}(n)$ corresponds closely to the number of representations of $n$ of the kind we are interested in.

In this paper we prove that the deviation of $J_{3}(n)$ from its expected main term is uniformly small for large moduli, namely in the following sense.

Theorem 1.1. For every $A, \varepsilon>0$, every sufficiently large $n$ and for $D \leq$ $n^{1 / 5-\varepsilon}$ we have

$$
\mathcal{E}:=\sum_{D<k \leq 2 D} \frac{k}{\varphi(k)} \sum_{\left(b_{1}, k\right)=1} \frac{1}{\varphi(k)} \sum_{b_{2}}\left|J_{3}(n)-\frac{n^{2}}{k^{2}} \mathcal{S}(n, k)\right| \ll \frac{n^{2}}{(\log n)^{A}} .
$$

Here $\mathcal{S}(n, k)$ denotes the singular series for this special Goldbach problem. In depends on $k$, while $J_{3}(n)$ depends on $k, b_{1}, b_{2}$; residue $b_{3}$ is simply $b_{3} \equiv n-b_{1}-b_{2}(k)$. Namely, see [4], for odd $n$ we have

$\mathcal{S}(n, k)=C(k) \prod_{p \mid k} \frac{p^{3}}{(p-1)^{3}+1} \prod_{\substack{p \mid n \\ p \nmid k}} \frac{(p-1)\left((p-1)^{2}-1\right)}{(p-1)^{3}+1} \prod_{p>2}\left(1+\frac{1}{(p-1)^{3}}\right)$,

where $p>2$ throughout, $C(k)=2$ for odd $k$ and $C(k)=8$ for even $k$.

As a consequence of Theorem 1.1, we prove in section 2 the following result.

Theorem 1.2. Let $A, \varepsilon>0$ and let $n \in \mathbb{N}$ be odd and sufficiently large. Then for all $k \leq R:=n^{1 / 5-\varepsilon}$ with at most $\ll R(\log n)^{-A}$ exceptions there exists a representation $n=p_{1}+p_{2}+p_{3}$ with primes $p_{i} \equiv b_{i}(k)$ for all but $\ll A(k)(\log n)^{-A}$ many admissible triplets $b_{1}, b_{2}, b_{3}$.

So there are few exceptions for $k$, and also the number of exceptions of admissible triplets is small compared with the number $A(k)$ of all admissible triplets.

Let us compare this Theorem 1.2 with the result of J. Liu and T. Zhang in [4] who show the assertion for $R:=n^{1 / 8-\varepsilon}$ and all admissible triplets. In another paper [3], Z. Cui improved this to $R:=n^{1 / 6-\varepsilon}$. Further C. Bauer and Y. Wang showed in [2] the assertion for $R:=n^{5 / 48-\varepsilon}$, but with only $\ll(\log n)^{B}$ many exceptions.

Here we improved the bound for $R$ again, but at the cost of possibly a few exceptions of admissible triplets. 


\section{Proof of Theorem 1.2}

First of all we give a lower bound for $A(k)$ :

Lemma 2.1. For odd $n$ we have $A(k) \gg \frac{\varphi(k)^{2}}{(\log k)^{3}}$. More precisely, for every reduced residue $b_{1} \bmod k$ there are $\gg \frac{\varphi(k)}{(\log k)^{3}}$ many reduced residues $b_{2}$ mod $k$ with $\left(n-b_{1}-b_{2}, k\right)=1$.

Proof. Fix a reduced residue $b_{1} \bmod k$. Now count the $b_{2}$ with $\left(b_{2}, k\right)=$ $\left(n-b_{1}-b_{2}, k\right)=1$. So $b_{2}$ is to be chosen such that for all prime divisors $p>2$ of $k$ we have $b_{2} \not \equiv 0(p)$ and $b_{2} \not \equiv n-b_{1}(p)$. This gives $\geq p-2$ possibilities for $b_{2} \bmod p$, and $\geq p^{l-1}(p-2)$ possiblilities for $b_{2} \bmod p^{l}$. If $p=2$ for even $k$ we have an odd $b_{1}$, so $n-b_{1}$ is even and therefore one can take $b_{2} \equiv 1(2)$, so there are $2^{\nu_{2}(k)-1}$ possibilities for $b_{2} \bmod 2^{\nu_{2}(k)}$, if $2^{\nu_{2}(k)} \| k$.

Therefore the number of $b_{2}$ is at least

$$
2^{\max \left\{0, \nu_{2}(k)-1\right\}} \prod_{\substack{p^{l} \| k \\ p \neq 2}} p^{l-1}(p-2)=\varphi(k) \prod_{\substack{p \mid k \\ p \neq 2}} \frac{p-2}{p-1}
$$

with

$$
\begin{aligned}
& \prod_{\substack{p \mid k \\
p \neq 2}} \frac{p-1}{p-2}=\prod_{\substack{p \mid k \\
p \neq 2}}\left(1+\frac{1}{p-2}\right) \leq \prod_{p \mid k}\left(1+\frac{2}{p-1}\right) \\
& \quad \leq \sum_{q=1}^{k} \frac{\mu(q)^{2} 2^{\omega(q)}}{\varphi(q)} \ll \sum_{q=1}^{k} \frac{\tau(q)}{q} \log k \ll(\log k)^{3} .
\end{aligned}
$$

Now we deduce Theorem 1.2 as a corollary of Theorem 1.1.

Fix $A, \varepsilon>0$ and let $n$ be odd and sufficiently large. Consider

$$
R_{3}(n):=\sum_{\begin{array}{c}
p_{1}, p_{2}, p_{3} \\
p_{1}+p_{2}+p_{3}=n \\
p_{i} \equiv b_{i}(k), \\
i=1,2,3
\end{array}} \log p_{1} \log p_{2} \log p_{3} \quad \text { and } \quad r_{3}(n):=\sum_{\begin{array}{c}
p_{1}, p_{2}, p_{3} \\
p_{1}+p_{2}+p_{3}=n \\
p_{i} \equiv b_{i}(k), \\
i=1,2,3
\end{array}} 1 .
$$

Let $D<k \leq 2 D$ with $D \leq R:=n^{1 / 5-\varepsilon}$. For any admissible triplet $b_{1}, b_{2}, b_{3} \bmod k$ we have

$$
\left|R_{3}(n)-J_{3}(n)\right| \leq(\log n)^{3} W_{3},
$$

where $W_{3}$ denotes the number of solutions of $p^{l}+q^{j}+r^{m}=n$ with $p, q, r$ prime, and where $l, j$ or $m$ are at least 2 such that $p^{l} \equiv b_{1}(k), q^{j} \equiv b_{2}(k)$ and $r^{m} \equiv b_{3}(k)$. 
Now we prove that

$$
\sum_{D<k \leq 2 D} k \max _{\substack{b_{1}, b_{2}, b_{3} \\ \text { admissible }}} W_{3} \ll \frac{n^{2}}{(\log n)^{A+3}}
$$

For this, we split the number $W_{3}$ according to whether at least two of the exponents $l, j, m$ are $\geq 2$ or only one is, and for this we write $W_{3}=$ $W_{1}+W_{2}$. There are at most $\sqrt{n}$ prime powers $\leq n$ with exponent $\geq 2$, so in the first case we have $W_{1} \ll n$, and the sum with $W_{1}$ replacing $W_{3}$ is $\ll D^{2} W_{1} \ll D^{2} n \ll \frac{n^{2}}{(\log n)^{A+3}}$.

In the second case, if only one exponent is $\geq 2$, we have $W_{2} \ll \sqrt{n} \cdot \frac{n}{k}=$ $\frac{n^{3 / 2}}{k}$, and so the sum with $W_{2}$ replacing $W_{3}$ is $\ll D n^{3 / 2} \ll \frac{n^{2}}{(\log n)^{A+3}}$.

So for $D \leq n^{1 / 5-\varepsilon}$ it follows from Theorem 1.1 that

$$
\begin{aligned}
\sum_{D<k \leq 2 D} & \frac{k}{\varphi(k)} \sum_{\left(b_{1}, k\right)=1} \frac{1}{\varphi(k)} \sum_{b_{2} \text { adm. }}\left|R_{3}(n)-\frac{n^{2}}{k^{2}} \mathcal{S}(n, k)\right| \\
\leq & \sum_{D<k \leq 2 D} \frac{k}{\varphi(k)} \sum_{\left(b_{1}, k\right)=1} \frac{1}{\varphi(k)} \sum_{b_{2} \text { adm. }}\left|R_{3}(n)-J_{3}(n)\right| \\
& +\sum_{D<k \leq 2 D} \frac{k}{\varphi(k)} \sum_{\substack{\left(b_{1}, k\right)=1 \\
\varphi(k)}} \frac{1}{b_{2} \text { adm. }}\left|J_{3}(n)-\frac{n^{2}}{k^{2}} \mathcal{S}(n, k)\right| \\
& \ll(\log n)^{3} \sum_{D<k \leq 2 D} k \max _{\substack{b_{1}, b_{2}, b_{3} \\
\text { admissible }}} W_{3}+\frac{n^{2}}{(\log n)^{A}} \\
\ll & \frac{n^{2}}{(\log n)^{A}} .
\end{aligned}
$$

So the formula of Theorem 1.1 holds also with $R_{3}(n)$ replacing $J_{3}(n)$.

Now for $D<k \leq 2 D$ we have $A(k):=\#\left\{b_{1}, b_{2}\right.$ admissible mod $\left.k\right\}$. Let $T(k):=\#\left\{b_{1}, b_{2}\right.$ admissible $\left.\bmod k ; R_{3}(n)=0\right\}$, consider the set

$$
\mathcal{K}_{D}:=\left\{k ; D<k \leq 2 D, T(k) \geq A(k)(\log n)^{-A}\right\}
$$

and let $K_{D}$ be its cardinality. 
Since $\mathcal{S}(n, k) \gg 1$ if it is positive, which is the case for admissible triplets and odd $n$ (see its formula above as an Euler product), we have

$$
\begin{aligned}
K_{D} \cdot \frac{n^{2}}{D} & \ll \sum_{\substack{D<k \leq 2 D \\
k \in \mathcal{K}_{D}}} \frac{k}{T(k)} \sum_{\substack{b_{1}, b_{2} \text { adm. } \\
R_{3}(n)=0}}\left|\frac{n^{2}}{k^{2}} \mathcal{S}(n, k)\right| \\
& \ll \sum_{D<k \leq 2 D} \frac{k}{A(k)} \sum_{\substack{b_{1}, b_{2} \text { adm. } \\
\text { adm }}}(\log n)^{A}\left|R_{3}(n)-\frac{n^{2}}{k^{2}} \mathcal{S}(n, k)\right| \\
& \ll(\log n)^{A+3} \sum_{D<k \leq 2 D} \frac{k}{\varphi(k)^{2}} \sum_{\substack{b_{1}, b_{2} \\
\text { adm. }}}\left|R_{3}(n)-\frac{n^{2}}{k^{2}} \mathcal{S}(n, k)\right| \ll \frac{n^{2}}{(\log n)^{A}},
\end{aligned}
$$

using Lemma 2.1 and the above. Therefore it follows that $K_{D} \ll D(\log n)^{-A}$, so for all $k \notin \mathcal{K}_{D}$ we have $R_{3}(n)>0$ for all but $\ll A(k)(\log n)^{-A}$ many admissible triplets $b_{1}, b_{2}, b_{3}$, and then $r_{3}(n) \gg R_{3}(n)(\log n)^{-3}$ is positive, too. This shows Theorem 1.2, since the overall number of exceptions is

$$
\ll \sum_{D=2^{i} \leq R} K_{D} \ll(\log n) \cdot \frac{R}{(\log n)^{A+1}}=\frac{R}{(\log n)^{A}} .
$$

\section{Proof of Theorem 1.1}

Our proof is in two steps using the circle method.

Let $A, \varepsilon, \theta>0, B \geq 2 A+1$ and $D \leq n^{1 / 4}(\log n)^{-\theta}$.

We define major arcs $\mathfrak{M} \subseteq \mathbb{R}$ by

$$
\left.\mathfrak{M}:=\bigcup_{q \leq D(\log n)^{B}} \bigcup_{\substack{0<a<q \\(a, q)=1}}\right] \frac{a}{q}-\frac{D(\log n)^{B}}{q n}, \frac{a}{q}+\frac{D(\log n)^{B}}{q n}[
$$

and minor arcs by

$$
\mathfrak{m}:=]-\frac{D(\log n)^{B}}{n}, 1-\frac{D(\log n)^{B}}{n}[\backslash \mathfrak{M} .
$$

For $\alpha \in \mathbb{R}$ and some residue $b \bmod k$ denote

$$
S_{b}(\alpha):=S_{b, k}(\alpha):=\sum_{\substack{m \leq n \\ m \equiv b(k)}} \Lambda(m) e(\alpha m)
$$

From the orthogonal relations for $e(\alpha m)$ it follows that

$$
J_{3}(n)=\int_{0}^{1} S_{b_{1}}(\alpha) S_{b_{2}}(\alpha) S_{b_{3}}(\alpha) e(-n \alpha) d \alpha
$$


By

$$
J_{3}^{\mathfrak{M}}(n):=\int_{\mathfrak{M}} S_{b_{1}}(\alpha) S_{b_{2}}(\alpha) S_{b_{3}}(\alpha) e(-n \alpha) d \alpha
$$

we denote the value of the integral for $J_{3}(n)$ on the major arcs $\mathfrak{M}$ and by

$$
J_{3}^{\mathfrak{m}}(n):=J_{3}(n)-J_{3}^{\mathfrak{M}}(n)
$$

its value on the minor arcs $\mathfrak{m}$.

We first consider the major arcs.

Theorem 3.1. For $D \leq n^{1 / 5-\varepsilon}$ we have

$$
\mathcal{E}^{\mathfrak{M}}:=\sum_{D<k \leq 2 D} k \max _{\substack{b_{1}, b_{2}, b_{3} \\ \text { admissible }}}\left|J_{3}^{\mathfrak{M}}(n)-\frac{n^{2}}{k^{2}} \mathcal{S}(n, k)\right| \ll \frac{n^{2}}{(\log n)^{A}} .
$$

We can give a very short proof of Theorem 3.1 simply by adapting the result of J. Liu and T. Zhang in [4] for the major arcs used here. In fact, by following their proof we see that for $P:=D(\log n)^{B}$ and $Q:=\frac{n}{D(\log n)^{B}}$ and any $U \leq P$, we have to choose $D$ such that the conditions

$$
\begin{aligned}
U & \leq n^{1 / 3}(\log n)^{-E}, & & (U Q)^{-1} \leq U^{-3}(\log n)^{-E} \\
D U & \leq D^{1 / 3-\delta} n^{1 / 3}(\log n)^{-E}, & & (U Q)^{-1} \leq D^{1-\delta}(D U)^{-3}(\log n)^{-E}
\end{aligned}
$$

are satisfied for any $E>0$ and small $\delta>0$. The optimal choice of $D$ is therefore given by $D \leq n^{1 / 5-\varepsilon}$. This proves Theorem 3.1. The improvement in this paper comes from the different intervals used as major and minor arcs such that working on the minor arcs with mean values over $b_{1}, b_{2}$ is still possible.

Specifically for an estimate on the minor arcs, we show the following in the next section.

Theorem 3.2. For $D \leq n^{1 / 4}(\log n)^{-\theta}$ we have

$$
\mathcal{E}^{\mathfrak{m}}:=\sum_{D<k \leq 2 D} \frac{k}{\varphi(k)} \sum_{\left(b_{1}, k\right)=1} \frac{1}{\varphi(k)} \sum_{\substack{\left(b_{2}, k\right)=1 \\ a d m .}}\left|J_{3}^{\mathfrak{m}}(n)\right| \ll \frac{n^{2}}{(\log n)^{A}} .
$$

Theorem 1.1 is then a corollary of Theorems 3.1 and 3.2 since $\mathcal{E} \leq$ $\mathcal{E}^{\mathfrak{M}}+\mathcal{E}^{\mathfrak{m}}$.

This Theorem is the interesting part of Theorem 1.1, where we can attain a higher power of $n$ for the bound of $D$ by considering the mean value over $b_{1}, b_{2}$ instead of the maximum. But because of this we have to allow exceptions of admissible triplets in Theorem 1.2, as we have seen in its proof.

In both Theorems 3.1 and 3.2 the resulting bound for $D$ is the optimum with the given method, so these bounds cannot be balanced to get a larger 
range than $n^{1 / 5}$. Also the cited method for the major arcs cannot be improved by using mean values over $b_{1}, b_{2}$ since the character sum estimates used are independent of $b_{1}, b_{2}$.

\section{Proof of Theorem 3.2: the estimate on the minor arcs}

Let $D \leq n^{1 / 4}(\log n)^{-\theta}$ and let $\sum^{*}$ denote a sum over reduced residues $\bmod k$. We have

$$
\begin{aligned}
\mathcal{E}^{\mathfrak{m}} & \ll \sum_{D<k \leq 2 D} \frac{k}{\varphi(k)^{2}} \sum_{b_{1}, b_{2}}^{*}\left|J_{3}^{\mathfrak{m}}(n)\right| \\
& \leq \sum_{D<k \leq 2 D} \frac{k}{\varphi(k)^{2}} \sum_{b_{1}, b_{2}}^{*} \int_{\mathfrak{m}}\left|S_{b_{1}}(\alpha) S_{b_{2}}(\alpha) S_{n-b_{1}-b_{2}}(\alpha)\right| d \alpha \\
& =\sum_{D<k \leq 2 D} \frac{k}{\varphi(k)} \sum_{b_{1}}^{*} \int_{\mathfrak{m}}\left|S_{b_{1}}(\alpha)\right| \cdot \frac{1}{\varphi(k)} \sum_{b_{2}}^{*}\left|S_{b_{2}}(\alpha) S_{n-b_{1}-b_{2}}(\alpha)\right| d \alpha \\
& \leq \sum_{D<k \leq 2 D} \frac{k}{\varphi(k)} \int_{\mathfrak{m}} \sum_{b_{1}}^{*}\left|S_{b_{1}}(\alpha)\right| \\
& \leq \sum_{D<k \leq 2 D} \frac{1}{\varphi(k)}\left(\sum_{b_{2}} \frac{k}{\varphi(k)} \operatorname{mad}_{\alpha \in \mathfrak{m}} \sum_{b_{1}}^{*}\left|S_{b_{2}}(\alpha)\right|^{2}\right)^{1 / 2}\left(\left.S_{b_{1}}(\alpha)\left|\frac{1}{\varphi(k)} \sum_{b_{2} \bmod k}\right| S_{n-b_{1}-b_{2}}(\alpha)\right|^{2}\right)^{1 / 2} d \alpha \\
& \left.\ll n(\log n)^{3} \sum_{D<k \leq 2 D} \frac{1}{\varphi(k)} \max _{\alpha \in \mathfrak{m}} \sum_{b_{1}}^{*}(\beta)\right|^{2} d \beta \\
& \leq n(\log n)^{3} \sum_{D<k \leq 2 D} \max _{\alpha \in \mathfrak{m}}\left(\frac{1}{\varphi(k)} \sum_{b_{1}}^{*}\left|S_{b_{1}}(\alpha)\right|^{2}\right)^{1 / 2} \\
& \ll n(\log n)^{3} \sum_{D<k \leq 2 D}\left(\frac{n^{2}}{\left.D^{2}(\log n)^{2 A+6}\right)^{1 / 2}} \cdot\right.
\end{aligned}
$$

In the last step we use Lemma 4.1 below, valid for $D \leq n^{1 / 4}(\log n)^{-\theta}$ and suitable chosen $\theta, B>0$ depending just on $A>0$.

Now the above is $\ll n(\log n)^{3} D \frac{n}{D(\log n)^{A+3}}=\frac{n^{2}}{(\log n)^{A}}$ as was to be shown for the minor arcs.

So what is left to show is the following.

Lemma 4.1. For all $A>0$ and $B \geq 2 A+1, \theta \geq B / 2$ let $D \leq n^{1 / 4}(\log n)^{-\theta}$ and $\alpha \in \mathbb{R}$ with $\left\|\alpha-\frac{u}{v}\right\|<\frac{1}{v^{2}}$ for some integers $u, v$ with $(u, v)=1$ and 
$D(\log n)^{B} \leq v \leq \frac{n}{D(\log n)^{B}}$. Then for $D<d \leq 2 D$ we have

$$
\frac{1}{\varphi(d)} \sum_{c,(c, d)=1}\left|S_{c, d}(\alpha)\right|^{2} \ll \frac{n^{2}}{D^{2}(\log n)^{A}}
$$

We remark that for $\alpha \in \mathfrak{m}$ there exist $u, v$ with $(u, v)=1, v \leq \frac{n}{D(\log n)^{B}}$ and $\left\|\alpha-\frac{u}{v}\right\|<\frac{D(\log n)^{B}}{v n} \leq \frac{1}{v^{2}}$ by Dirichlet's approximation theorem, so $v \geq D(\log n)^{B}$ since $\alpha \in \mathfrak{m}$, and therefore the conditions of Lemma 4.1 are satisfied.

For the proof we need the following auxiliary Lemma. (See [5], Lemma 4.11 and 4.10.)

Lemma 4.2. Let $\left\|\alpha-\frac{u}{v}\right\| \leq \frac{1}{v^{2}},(u, v)=1$. Then

(a) $\quad \sum_{m \leq X} \min \left(Y,\|\alpha m\|^{-1}\right) \ll \frac{X Y}{v}+Y+(X+v)(\log v)$,

(b) $\quad \sum_{m \leq X} \min \left(\frac{Y}{m},\|\alpha m\|^{-1}\right) \ll\left(\frac{Y}{v}+X+v\right) \log (2 v X)$

Proof of Lemma 4.1. Fix $n$ large and $D \leq n^{1 / 4}(\log n)^{-\theta}$, and let $\alpha, u$ and $v$ be as given in Lemma 4.1.

We apply Vaughan's identity on the exponential sum $S_{c, d}(\alpha)$, see for example A. Balog in [1], where a similar Lemma is given (Lemma 2 there). From that it follows that it suffices to show for any complex coefficients $\left|a_{m}\right|,\left|b_{k}\right| \leq 1$ and any $M \in \mathbb{N}$ with

$$
\begin{aligned}
& I: \quad M \leq V^{2}, \text { if } b_{k}=1 \text { for all } k, \\
& I I: \quad V \leq M \leq \frac{n}{V} \text { else, where } V:=D(\log n)^{B},
\end{aligned}
$$

we have

$$
\sum_{(c, d)=1}\left|\sum_{m \sim M} \sum_{\substack{k \leq n / m \\ k m \equiv c(d)}} a_{m} b_{k} e(\alpha m k)\right|^{2} \ll \frac{n^{2}}{D(\log n)^{A}} .
$$

Here $m \sim M$ means $M<m \leq M^{\prime}$ for some $M^{\prime} \leq 2 M$ 
We consider first case II: Then the left hand side becomes (where $m^{*}$ denotes the inverse of $m \bmod d$ ):

$$
\begin{aligned}
& I I:=\sum_{(c, d)=1}\left|\sum_{\substack{m \sim M \\
m, d)=1}} a_{m} \sum_{\substack{k \leq n / m \\
k m \equiv c(d)}} b_{k} e(\alpha m k)\right|^{2} \\
& \leq \sum_{(c, d)=1} M \sum_{\substack{m \sim M \\
(m, d)=1}}\left|\sum_{\substack{k \leq n / m \\
k \equiv c m^{*}(d)}} b_{k} e(\alpha m k)\right|^{2} \\
& =M \sum_{\substack{m \sim M \\
(m, d)=1}} \sum_{(c, d)=1}\left|\sum_{\substack{k \leq n / m \\
k \equiv c(d)}} b_{k} e(\alpha m k)\right|^{2} \\
& =M \sum_{m \sim M} \sum_{(c, d)=1} \sum_{\substack{k \leq n / m \\
k \equiv c(d)}} b_{k} \sum_{\begin{array}{c}
k^{\prime} \leq n / m \\
k^{\prime} \equiv k(d)
\end{array}} \overline{b_{k^{\prime}}} e\left(\alpha m\left(k-k^{\prime}\right)\right) \\
& =M \sum_{m \sim M} \sum_{\substack{k \leq n / m \\
(k, d)=1}} b_{k} \sum_{\substack{k^{\prime} \leq n / m \\
k^{\prime} \equiv k(d)}} \overline{b_{k^{\prime}}} e\left(\alpha m\left(k-k^{\prime}\right)\right) \\
& =M \sum_{m \sim M} \sum_{\substack{k \leq n / m \\
(k, d)=1}} b_{k} \sum_{\substack{l \geq(k-n / m) / d \\
l \leq(n / m-1) / d}} \overline{b_{k-l d}} e(\alpha m l d) \\
& \leq M \sum_{k \leq n / M} \sum_{|l| \leq n / M d}\left|\sum_{\substack{m \sim M \\
m \leq n / k \\
m \leq n / \max \{k-l d, l d+1\}}} e(\alpha m l d)\right| .
\end{aligned}
$$

Now the absolute value of the exponential sum is $\ll \min \left(M,\|\alpha l d\|^{-1}\right)$, so

$$
\begin{aligned}
I I & \ll M \frac{n}{M} \sum_{|l| \leq n / M d} \min \left(M,\|\alpha l d\|^{-1}\right) \\
& \ll n \sum_{\substack{L \leq n / M \\
d \mid L}} \min \left(M,\|\alpha L\|^{-1}\right)+n M \\
& \leq n\left(\sum_{\substack{d \leq n / M \\
d \mid L}} 1^{2}\right)^{1 / 2}\left(\sum_{L \leq n / M} M \min \left(M,\|\alpha L\|^{-1}\right)\right)^{1 / 2}+n M \\
& \ll n\left(\frac{n}{M d}\right)^{1 / 2} M^{1 / 2}\left(\frac{n}{v}+M+\left(\frac{n}{M}+v\right)(\log n)\right)^{1 / 2}+n M,
\end{aligned}
$$


because of the auxiliary Lemma 4.2 (a). So expression $I I$ is $\ll \frac{n^{2}}{D(\log n)^{A}}$ since we have $D(\log n)^{B}=V \leq M \leq n / V$ in case II, and since $D(\log n)^{B} \ll$ $v \ll \frac{n}{D(\log n)^{B}}$ for $B \geq 2 A+1$.

Now consider case I: Then the left hand side becomes (again $m^{*}$ denotes the inverse of $m \bmod d$ ):

$$
\begin{aligned}
& I:=\sum_{(c, d)=1}\left|\sum_{\substack{m \sim M \\
m, d)=1}} a_{m} \sum_{\substack{k \leq n / m \\
k m \equiv c(d)}} e(\alpha m k)\right|^{2} \\
& \leq \sum_{(c, d)=1} M \sum_{\substack{m \sim M \\
(m, d)=1}}\left|\sum_{\substack{k \leq n / m \\
k \equiv c m^{*}(d)}} e(\alpha m k)\right|^{2} \\
& \leq M \sum_{m \sim M} \sum_{(c, d)=1}\left|\sum_{\substack{k \leq n / m \\
k \equiv c(d)}} e(\alpha m k)\right|^{2} \\
& =M \sum_{m \sim M} \sum_{(c, d)=1} \sum_{\substack{k \leq n / m \\
k \equiv c(d)}} e(\alpha m k) \sum_{\substack{k^{\prime} \leq n / m \\
k \equiv k^{\prime}(d)}} e\left(-\alpha m k^{\prime}\right) \\
& =M \sum_{m \sim M} \sum_{\substack{k \leq n / m \\
(k, d)=1}} \sum_{\substack{k^{\prime} \leq n / m \\
k \equiv k^{\prime}(d)}} e\left(\alpha m\left(k-k^{\prime}\right)\right) \\
& \leq M \sum_{m \sim M} \sum_{k \leq n / m}\left|\sum_{l \geq(k-n / m) / d} e(\alpha m d l)\right| \\
& \ll M \sum_{m \sim M} \sum_{k \leq n / M}\left(\min \left(\frac{n}{m d},\|\alpha m d\|^{-1}\right)+1\right) \\
& \ll n \sum_{m \sim M} \min \left(\frac{n}{m d},\|\alpha m d\|^{-1}\right)+M n \\
& \ll n \sum_{L \sim M d} \min \left(\frac{n}{L},\|\alpha L\|^{-1}\right)+M n \\
& \ll n\left(\frac{n}{v}+M d+v\right)(\log n)+M n,
\end{aligned}
$$

using auxiliary Lemma 4.2 (b). Now we get $I \ll \frac{n^{2}}{D(\log n)^{A}}$ since $D(\log n)^{B} \ll$ $v \ll \frac{n}{D(\log n)^{B}}$ with $B \geq A+1$ and since $M d \ll V^{2} d \ll D^{3}(\log n)^{B} \ll$ $\frac{n}{D(\log n)^{B}}$ for $D \leq n^{1 / 4}(\log n)^{-\theta}$ and $\theta \geq B / 2$. So Lemma 4.1 is shown. 
Final remark. As was kindly pointed out to me by Z. Cui, it is possible to improve the statement on the major arcs such that Theorems 1.1, 3.1 and 3.2 hold for the improved exponent $1 / 4$ instead of $1 / 5$. The idea for this major arc improvement comes from Z. Cui in [3].

\section{References}

[1] A. Balog, The Prime $k$-Tuplets Conjecture on Average. Analytic number theory, Proc. Conf. in Honor of Paul T. Bateman, Urbana/IL (USA), 1989, Prog. Math. 85 (1990), 4775 .

[2] C. Bauer, Y. WAng, On the Goldbach conjecture in arithmetic progressions. Rocky Mountain J. Math. 36 (1) (2006), 35-66.

[3] Z. CuI, The ternary Goldbach problem in arithmetic progression II. Acta Math. Sinica (Chin. Ser.) 49 (1) (2006), 129-138.

[4] J. Liu, T. Zhang, The ternary Goldbach problem in arithmetic progressions. Acta Arith. 82 (3) (1997), 197-227.

[5] M. B. Nathanson, Additive Number Theory: The Classical Bases. Graduate texts in Mathematics 164, Springer-Verlag, 1996.

Karin HALUPCZOK

Albert-Ludwigs-Universität Freiburg

Eckerstr. 1

D-79104 Freiburg, Allemagne

E-mail: karin.halupczok@math.uni-freiburg.de

$U R L$ : http://home.mathematik.uni-freiburg.de/halupczok/ 